\title{
APROXIMACIÓN A UN CONCEPTO DE CALIDAD DE VIDA EN LA VEJEZ. Escuchando a las personas mayores.
}

\author{
Paulina Osorio ${ }^{1}$ /María José Torrejón ${ }^{2}$ /Natalia Vogeß
}

A partir de los ańos setenta, el estudio de la calidad de vida, desde una perspectiva biomédica, psicológica y social, comienza a hacerse más sistemático y relevante en el mundo académico. La calidad de vida se la ha definido, principalmente desde un punto de vista subjetivo (OMS, 1995), destacando la percepción de las personas sobre diversos ámbitos de sus vidas, donde lo importante es destacar la relevancia del contexto social y cultural y del sistema de valores en el que experimentan su vida cotidiana.

En cuanto al estudio y la medición de la calidad de vida en la vejez, se ha puesto el énfasis en los aspectos biomédicos de la vida de las personas. Sin embargo, la revisión de bibliografía en el tema muestra que hay otros factores importantes, tales como: grados de autonomía, interacciones y redes sociales, condiciones económicas, vivienda y entorno urbano, actividades recreativas y de ocio, relación con cuidadores y capacidad de tomar decisiones al final de la vida, e incluso, la misma dimensión biográfica, influirían en la percepción de calidad de vida.

Antropóloga Social, Universidad de Chile. Doctora en Sociología, Universidad del País Vasco, España. Profesora del Departamento de Antropología, Facultad de Ciencias Sociales de la Universidad de Chile e investigadora del Programa Domeyko en Salud de la Universidad de Chile. posorio@uchile.cl

2 Antropóloga Social, Universidad de Chile. Magíster en Antropología y Desarrollo, Universidad de Chile. Investigadora Programa PULSO, Facultad de Ciencias Sociales y del Programa Domeyko en Salud de la Universidad de Chile.

3 Socióloga, Universidad de Concepción. Magíster @ en Antropología y Desarrollo, Universidad de Chile. Investigadora Programa PULSO, Facultad de Ciencias Sociales y del Programa Domeyko en Salud de la Universidad de Chile. 
En este marco de antecedentes se está desarrollando una investigación titulada "Calidad de vida y adulto mayor" ${ }^{4}$, la cual plantea la hipótesis de que existen otros factores gravitantes en la calidad de vida del adulto mayor que son tan importantes como los biomédicos, pues hacen referencia a aspectos socioculturales particulares no sólo de un grupo etáreo, sino también, de una cultura. Al aplicar instrumentos elaborados en otros países, se comete el error de extrapolar resultados que pueden no ser aplicables a una determinada realidad social. El objetivo general de la investigación es determinar la importancia relativa de los factores sociales, culturales, económicos y biomédicos en la calidad de vida de los adultos mayores residentes en Santiago, para lo cual, se han definido los siguientes objetivos específicos: (a) Construir un concepto de calidad de vida desde la perspectiva de los propios adultos mayores; (b) Determinar la importancia relativa de las dimensiones salud física, salud psicológica, bienestar espiritual, relaciones sociales y ambiente (incluidos aspectos económicos) en grupos de adultos mayores; (c) Diseñar una metodología y un instrumento de medición para ser aplicado en adultos mayores de Santiago.

La estrategia metodológica se estructura en base a dos etapas: una cualitativa y una cuantitativa. El desarrollo de la etapa cualitativa de la investigación ha contemplado el trabajo con dos técnicas de producción de datos cualitativos: grupos focales y entrevistas en profundidad. En el presente trabajo se describirá la primera de estas etapas y se darán a conocer los primeros resultados obtenidos a través de la realización de los grupos focales.

El grupo focal es una técnica de producción de datos cualitativos que permite realizar una discusión grupal organizada alrededor de una temática específica. Se trata de un grupo artificial pues, aunque los integrantes puedan conocerse de antemano, el grupo conformado en torno a un tema seleccionado no existe ni antes ni después de la sesión de conversación (Álvarez-Gayou Jurgenson, 2007). Es decir, aunque antes los integrantes del grupo hayan compartido instancias de conversación, es altamente im-

Investigación financiada por la Vicerrectoría de Investigación y Desarrollo de la Universidad de Chile, y que se inscribe al interior del Programa Domeyko en Salud (2007-2010). 
probable que lo hayan hecho en torno al problema que determina la conformación del grupo con fines investigativos, en nuestro caso, en torno a su calidad de vida.

Los estudios cualitativos trabajan con muestreos intencionales, es decir, que no siguen las leyes del azar. Esto significa que el investigador es quien selecciona las unidades de la muestra. La definición de nuestra muestra siguió las orientaciones del muestreo teórico - una de las modalidades del muestreo intencional-, cuyo principio básico es generar teoría por medio de la recolección, codificación y análisis de datos, en base a los cuales se va decidiendo los datos que se continuarán produciendo y en dónde encontrarlos. La idea, por lo tanto, ha sido profundizar en las categorías que se desea estudiar y producir la información más relevante para el concepto o teoría que se está elaborando: calidad de vida en personas mayores. El muestreo se da por finalizado cuando se alcanza el nivel de saturación, esto es, cuando dejan de aparecer nuevos conceptos y categorías relevantes. Las unidades o dimensiones elegidas son aquellas que garantizan mejor cantidad y calidad de la información. En definitiva, este tipo de muestreo permite que la muestra pueda ser alterada durante el proceso de producción de la información (Ruiz Olabuénaga, 1999).

Para la conformación de los grupos de discusión y las entrevistas en profundidad se definieron los siguientes criterios de selección:

\begin{tabular}{|l|l|}
\hline a) Sexo & $>$ Masculino \\
\hline b) Edad & $>$ Femenino \\
\hline \multirow{2}{*}{ Nivel socioeconómico (NSE) } & $>$ Tramo 1:60 a 75 años \\
& $>$ Alto \\
\hline \multirow{2}{*}{ civel de Autonomía } & $>$ Bajo \\
\hline d) Aús \\
\hline
\end{tabular}

Por lo tanto, los grupos focales han quedado conformados de la siguiente forma:

Grupos focales mixtos. Los integrantes fueron de ambos sexos, de diferente nivel socioeconómico y de ambos tramos etáreos. 
Grupos focales con integrantes seleccionados, que se conformaron según las variables indicadas.

En total se realizaron 16 grupos focales, con un promedio de 7 integrantes cada uno.

\section{Resultados preliminares}

La definición de calidad de vida en el adulto mayor, a la luz de los resultados preliminares de los grupos focales, se caracteriza por ser multidimensional y, a juicio de los/as entrevistados/as, subjetiva. Dicha subjetividad debe ser entendida desde dos perspectivas: la evaluación de la situación actual (vejez), asumiendo las limitaciones y posibilidades, y la satisfacción frente a dimensiones concretas de calidad de vida (objetivas), satisfacción que encuentra sus puntos de referencia en el contexto social y cultural en que el sujeto de desenvuelve.

\section{Cuadro No 1}

Dimensiones de la Calidad de Vida:

definición según percepciones de las personas mayores

\begin{tabular}{|c|c|c|c|}
\hline \multicolumn{4}{|c|}{ Objetivo } \\
\hline Social & Integración & Oportunidades & Seguridad \\
\hline Entorno físico & Movilidad & Habitabilidad & Naturaleza \\
\hline Individual & Autonomía & Familia & Rutina \\
\hline \multicolumn{4}{|c|}{ CONDICIONES DE VIDA } \\
\hline \multicolumn{4}{|l|}{ NIVEL DE VIDA } \\
\hline Material & Vivienda & Acceso salud & Alimento \\
\hline \multicolumn{4}{|c|}{ SALUD } \\
\hline
\end{tabular}

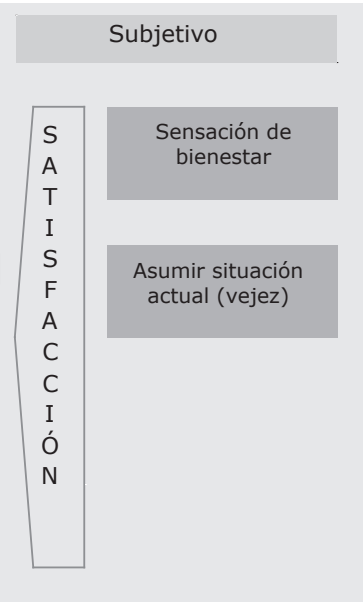


Los participantes de los grupos focales identifican la existencia de un nivel minimo necesario para tener una buena calidad de vida, dicho nivel se vincula a necesidades básicas materiales como tener una vivienda, acceso a salud y una buena alimentación. En la base de todas las dimensiones se encuentra la salud tanto mental como física y social, distinción realizada por los participantes de los grupos focales. Una buena calidad de vida desde la perspectiva de la salud se daría no por la ausencia de enfermedades, sino más bien por no ser "achacoso", es decir, por la percepción personal de una buena salud ("sentirse bien") y su reflejo a través de una buena actitud en las interacciones con otras personas. De ahí la mención de la salud social, vinculada a la mantención y creación de redes sociales para no caer en la soledad del hogar.

Dentro de las dimensiones objetivas también pueden distinguirse aquellas condiciones individuales y del entorno físico y social que facilitan o dificultan la calidad de vida. Las dimensiones más señaladas por los participantes guardan relación con lo individual (mantención de la autonomía y una rutina, y la relación con su familia) y el entorno social, especialmente en lo que refiere a las condiciones que genera (fundamentalmente el gobierno) para ellos: políticas públicas, consideración de sus necesidades, espacios de reunión, entre otras. El entorno social también incluye las relaciones intergeneracionales, particularmente la relación de respeto que las personas mayores esperan de parte de los jóvenes. Las personas que participan en grupos de adultos mayores dan mayor importancia a la mantención de actividades que llenen su día y les permita mantenerse en relación con sus pares. En tal sentido, asignan un papel central a la pertenencia a estos grupos como medio de mejoramiento de su calidad de vida.

La relación con la familia también se presenta como parte importante de los factores que permiten tener una buena calidad de vida. Dicha relación, para que resulte beneficiosa, debe estar marcada por las buenas distancias, esto significa que las personas mayores no quieren sentirse utilizadas por sus hijos para cuidar a sus nietos, pues ya dan su tarea de crianza por cumplida. 
Con respecto al entorno físico, se mencionan aspectos de la ciudad, más que de sus propios hogares. Aparece principalmente el transporte, específicamente los efectos que ha tenido el Transantiago en su rutina diaria, el cual los ha llevado a relegarse en sus casas por la incomodidad del servicio, el desconocimiento de los recorridos y la incertidumbre ante el funcionamiento del sistema. En general, se menciona la agresividad de la ciudad de Santiago para el adulto mayor, vinculándose lo concerniente al entorno físico con el entorno social, las dificultades para movilizarse y caminar por la ciudad para realizar sus actividades cotidianas y acceder a los servicios que requieren.

El reconocimiento de las dimensiones que componen la calidad de vida y su preponderancia cambia según el nivel socioeconómico al que pertenecen los participantes de los grupos focales, así como el género de éstos. Los grupos conformados por personas mayores pertenecientes a NSE Alto ponen su atención en la realización de actividades recreativas y la valoración del tiempo de ocio, mientras que las personas de NSE medio y bajo se centran principalmente en aspectos económicos, dado que el acceso a la salud, alimentación adecuada, actividades recreativas, entre otras, son mediadas por el dinero. Este punto de atención es aún más notorio en los varones de estos estratos socioeconómicos.

El trabajo se reconoce como importante para tener una buena calidad de vida, especialmente en varones de NSE medio y bajo, pues reporta ingresos extra a las pensiones, además de permitirles mantener relaciones con otras personas. Sin embargo, se hace hincapié en que debe ser un trabajo realizado por gusto, pues a diferencia de casi todos los ámbitos o actividades realizadas a lo largo de la vida, la etapa de la vejez se distingue por ser un período en que se pueden elegir las actividades a realizar, optando por aquello que se hará por agrado y no por deber y obligación, como han experimentado diversas actividades y responsabilidades en otros periodos de sus vidas.

Con fines operativos para realizar el análisis se han ordenado las dimensiones y los temas vinculados, según se indica en la siguiente tabla: 


\section{Tabla No 1: dimensiones de la calidad de vida}

\begin{tabular}{|c|c|}
\hline Dimensiones & Definición \\
\hline Bienestar & $\begin{array}{l}\text { Percepción subjetiva de bienestar general. Incluye la percepción de buena } \\
\text { salud, la percepción frente a la vida, el desarrollo personal, etc. }\end{array}$ \\
\hline Viudez & $\begin{array}{l}\text { Percepción de la viudez como estado actual o posible, implicancias que } \\
\text { tiene. }\end{array}$ \\
\hline Solidaridad & $\begin{array}{l}\text { Aporte que se realiza a otros. Mantener la vigencia a partir de la posibilidad } \\
\text { de entregar algo a alguien, ya sea material, experiencia, etc. }\end{array}$ \\
\hline Autonomía & $\begin{array}{l}\text { Espacios, actividades y ámbitos reconocidos como importantes por las } \\
\text { personas mayores que influyen o facilitan su autonomía, incluye aquellos } \\
\text { valorados como indicadores de autonomía. }\end{array}$ \\
\hline Vida espiritual & $\begin{array}{l}\text { Valoración de aspectos vinculados a la vida espiritual, puede o no guardar } \\
\text { relación con una religión particular. }\end{array}$ \\
\hline Recreación & Actividades que se realizan en el tiempo libre para entretenerse. \\
\hline Futuro & Perspectiva del adulto mayor en Chile a mediano y largo plazo. \\
\hline Economía & $\begin{array}{l}\text { Aspectos vinculados a la economía personal y familiar que se ven afectados } \\
\text { durante la vejez o que resultan importantes para mantener un nivel aceptable } \\
\text { de calidad de vida. }\end{array}$ \\
\hline Trabajo & $\begin{array}{l}\text { Razones, motivaciones y posibilidades de inserción laboral de las personas } \\
\text { mayores. }\end{array}$ \\
\hline Salud & $\begin{array}{l}\text { Incluye tanto factores que influyen en la salud física, mental y social de las } \\
\text { personas mayores, como condiciones. }\end{array}$ \\
\hline Vivienda & $\begin{array}{l}\text { Condiciones físicas y familiares presentes en los hogares que facilitan/ } \\
\text { dificultan las actividades cotidianas de las personas mayores. Incluye aspectos } \\
\text { simbólicos y de significación. }\end{array}$ \\
\hline Físico & $\begin{array}{l}\text { Condiciones físicas, ambientales y urbanísticas que influyen en las actividades } \\
\text { cotidianas y en la posibilidad de mejorar la calidad de vida de las personas } \\
\text { mayores. }\end{array}$ \\
\hline Nacional & $\begin{array}{l}\text { Condiciones en Chile que facilitan/dificultan la calidad de vida del adulto } \\
\text { mayor. Incluye principalmente la percepción de las políticas públicas dirigidas } \\
\text { al adulto mayor. }\end{array}$ \\
\hline Género & $\begin{array}{l}\text { Diferencias establecidas por el género en el proceso de envejecimiento y la } \\
\text { etapa de la vejez. }\end{array}$ \\
\hline Entorno social & $\begin{array}{l}\text { Entorno social y familiar con el cual interaccionan las personas mayores y que } \\
\text { influye en sus actividades y percepción de calidad de vida. }\end{array}$ \\
\hline Participación & $\begin{array}{l}\text { Espacios e instancias en que las personas mayores pueden participar y/o } \\
\text { integrarse a la sociedad. }\end{array}$ \\
\hline
\end{tabular}


Las dimensiones descritas se componen a su vez de diversas temáticas específicas. La etapa de análisis profundizará en su identificación, con el propósito de reconocer los principales factores asociados a la calidad de vida, así como la elaboración de una definición según las características particulares de la población de adultos mayores de la ciudad de Santiago.

Los resultados preliminares dan cuenta de la complejidad de este concepto, caracterizado por su multidimensionalidad y conjugación de perspectivas objetivas y subjetivas para su definición. Lo anterior nos ha llevado a una aproximación de la calidad de vida entendida como calidades de vida, que profundizaremos con la realización de la etapa cuantitativa de la investigación.

\section{Bibliografía}

Álvarez-Gayou Jurgenson, J.L. (2007). Cómo hacer investigación cualitativa: Fundamentos y metodología. México: Paidós Educador.

OMS (1995). The World Health Organization Quality of life assessment (WHOQOL). Position Paper from the World Health Organization. Soc. Sci. Med. Whoqol Group 1995, 41(10):1403-1409.

Ruiz Olabuénaga, J.I. (1999). Metodología de la Investigación Cualitativa. Bilbao: Universidad de Deusto. 